\title{
2-去氢表雄酮苯亚甲基衍生物的合成及抗炎活性研究
}

\author{
朱 丽 ${ }^{a, b}$ 杨艳秋 ${ }^{c}$ 高佩佩 ${ }^{a, b}$ 安 雪 $a, b$ 孙莹莹 ${ }^{a, b}$ \\ 孙晓雯 $a, b$ 侯 悦*,c 单丽红*,a,b

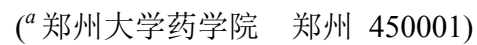 \\ $\left({ }^{b}\right.$ 新药创制与药物安全性评价河南省协同创新中心 郑州 450001) \\ ( ${ }^{c}$ 东北大学生命科学与健康学院 沈阳 110819)
}

\begin{abstract}
摘要 阿尔茨海默症作为 21 世纪全球主要健康危机之一, 由于其发病机制的复杂性, 目前还未找到很好的解决办法. 为了找到更加经济有效的治疗神经退行性疾病的药物, 合成了一系列新型 2-去氢表雄酮苯亚甲基衍生物, 并研究了其 对脂多糖(LPS)激活小胶质细胞株 BV-2 NO 释放及细胞存活率的影响. 结果表明所合成化合物作用 $24 \mathrm{~h}$ 后, 对 LPS 活 化的小鼠小胶质细胞株 BV2 的 NO 释放均有不同程度的抑制作用, 且具有一定的浓度依赖性. 活性最好的化合物 15 $\beta, 16 \beta$-亚甲基雄甾-2-(3-氯)-苯亚甲基-4,6-二烯-3,17-二酮(5a)和 15 , , 6 -亚甲基雄甾-2-(3,4,5-三甲氧基)-苯亚甲基-4,6二烯-3,17-二酮 $(\mathbf{5 j})$ 的 $\mathrm{IC}_{50}$ 值分别为 2.69 和 $3.28 \mu \mathrm{mol} \cdot \mathrm{L}^{-1}$, 优于阳性对照米诺环素. 此类化合物在涉及活化小胶质细胞 的神经退行性疾病方面的作用值得进一步研究.
\end{abstract}

关键词 去氢表雄酮; 查尔酮类似物; 阿尔茨海默症; 小胶质细胞

\section{Synthesis and Anti-inflammatory Activity Evaluation of 2-Dehydroepiandrosterone Benzene Methyl Derivatives}

\author{
Zhu, $\mathrm{Li}^{a, b} \quad$ Yang, Yanqiu ${ }^{c} \quad$ Gao, Peipei ${ }^{a, b} \quad$ An, Xue $e^{a, b} \quad$ Sun, Yingying ${ }^{a, b}$ \\ Sun, Xiaowen ${ }^{a, b}$ Hou, Yue ${ }^{*, c}$ Shan, Lihong ${ }^{*, a, b}$ \\ ( ${ }^{a}$ School of Pharmaceutical Sciences, Zhengzhou 450001) \\ $\left({ }^{b}\right.$ Collaborative Innovation Center of New Drug Research and Safety Evaluation, Zhengzhou 450001) \\ ( ${ }^{c}$ College of Life and Health Sciences, Northeastern University, Shenyang 110819)
}

\begin{abstract}
Alzheimer's Disease (AD) is one of the major health crises in the 21st century, and due to the complexity of its pathogenesis, there has no good solution to cure this disease. In order to find more economical and effective drugs for the treatment of neurodegenerative diseases, a series of novel 2-dehydroepiandrosterone benzathine derivatives were synthesized and their activity on the NO release of microglia cell line BV-2 activated by lipopolysaccharide (LPS), as well as their effect on cell viability were studied. The structures of the synthesized compounds were confirmed by ${ }^{1} \mathrm{H} N \mathrm{NMR},{ }^{13} \mathrm{C}$ NMR and HRMS. The results showed that all compounds had inhibitory effects on the NO release of LPS-activated mouse microglial cell line $\mathrm{BV} 2$ after $24 \mathrm{~h}$ of treatment, and it was dose-dependent. In particular, the $\mathrm{IC}_{50}$ values of 2-(3-chloro)benzylidene-15 $\beta, 16 \beta$ methylene-androstane-4,6-diene-3,17-dione (5a) and 2-(3,4,5-trimethoxy)benzylidene-15 $\beta, 16 \beta$-methylene-androstane-4,6-diene-3,17-dione $(\mathbf{5 j})$ were 2.69 and $3.28 \mu \mathrm{mol} \cdot \mathrm{L}^{-1}$, respectively, which were better than the positive control Minocycline. The results showed that these compounds may be effective in the development of neurodegenerative diseases involving activation of microglia, and the mechanism deserved further study.
\end{abstract}

Keywords dehydroepiandrosterone; chalcone analogue; alzheimer's disease; microglia

阿尔茨海默症(AD)是一种起病隐匿的进行性发展 的神经系统退行性疾病. 根据《2018 年世界阿尔茨海默
病报告》估计，目前全球有 5000 万 $\mathrm{AD}$ 患者; 据预测，到 2030 年, 患者数将增至 8200 万，至 2050 年，患者人数

\footnotetext{
* Corresponding authors. E-mail: shlh@zzu.edu.cn; houyue@mail.neu.edu.cn

Received January 25, 2019; revised March 22, 2019; published online April 11, 2019.

Project supported by the National Natural Science Foundation of China (Nos. 21402178, U1603125) and the Scientific Research Fund of Henan Province (No. 132300410195).

国家自然科学基金(Nos. 21402178, U1603125)和河南省科学研究基金(No.132300410195)资助项目
} 
将达到 1.52 亿. 仅 2018 年一年, 预计将有 1000 万新增 $\mathrm{AD}$ 患者. 据估算, 2018 年全球因 $\mathrm{AD}$ 产生的社会经济负 担约为 1 万亿美元, 到 2030 年, 该数字将增至 2 万亿美 元. 因此, 与神经退行性疾病相关的 $\mathrm{AD}$ 是 21 世纪全球 主要健康危机之一. 目前导致该疾病的确切机制仍不明 确, 认为导致 $\mathrm{AD}$ 的病理生理因素主要是乙酰胆碱酯酶 的缺乏、氧化应激、炎症、 $\beta$ 样淀粉蛋白沉积、 tau 蛋白 聚合和生物金属的稳态失衡 ${ }^{[1]}$. 其中小胶质细胞活化介 导的慢性炎症反应是神经退行性疾病的发生、发展过程 中的重要环节, 抑制小胶质细胞的激活可能成为药物发 现的一个新的靶点 ${ }^{[2]}$.

神经退行性疾病发病和治疗的复杂性使我们迫切 需要设计多靶向配体来解决这些疾病所涉及的互补途 径 ${ }^{[3]}$. 由于含查尔酮类结构(苯亚甲基、查尔酮、类黄酮、 异黄酮等)的各种化合物易于合成且产率较高, 以及广 泛的生物活性而备受关注, 尤其是抗菌、抗炎、抗癌、 抗氧化、神经保护、良好的脑通透性以及 AchE 抑制 剂 ${ }^{[4]}$. 例如, 含查尔酮结构的天然产物姜黄素可通过显 著保护神经元免于受小胶质细胞介导的炎症反应引起 的损伤而表现出良好的抗 $\mathrm{AD}$ 作用 ${ }^{[5]}$. 而且有研究表明, 查尔酮已被用于开发具有抗 5 -脂氧合酶和 $\beta$ 样淀粉蛋白 沉积的强效活化的新疗法, 说明查尔酮衍生物可作为针 对多重耐药的 $\mathrm{AD}$ 治疗的多功能候选物 ${ }^{[6]}$.

另一方面, 含有甾体结构的化合物对 $\mathrm{AD}$ 症的治疗 也有积极的影响. 如雌激素可通过外围效应和局部效 应, 多环节多途径地对阿尔茨海默病产生神经保护作 用, 对炎性因子的产生也具有抑制作用 ${ }^{[7]}$, 但由于其长 期服用所导致的不良反应而受限制. $\mathrm{AD}$ 患者大脑淀粉 样斑块的存在可以解释神经可塑性的改变, 四氢孕酮 (ALLO) 可以恢复海马依赖的学习记忆和神经祖细胞的 存活, 表明它可以用于治疗阿尔兹海默病 ${ }^{[8]}$. 作为神经 甾体类物质, 去氢表雄酮及其硫酸酯在脑中的浓度高于 其在血液中的浓度, 为了进一步增强此类物质穿透血脑 屏障的能力, 我们在其 15 和 16 位引入三元碳环结构, 同时在其 2 位引入苯亚甲基结构, 形成类似查尔酮的结 构单元, 初步测定了这些化合物对 LPS 活化小胶质细胞 释放 NO 作用的影响. 目标化合物的合成路线如 Scheme 1 所示.

\section{1 结果与讨论}

\section{1 目标化合物的合成}

以市售的去氢表雄酮(DHEA)为原料, 利用生物转 化的方法获得 7 $\alpha, 15 \alpha$-双羟基衍生物 2. 中间体 $\mathbf{2}$ 与三甲 基碘化亚砜在碱性条件下通过硫叶立德反应得到中间 体 3, 然后以甲苯作溶剂, 中间体 $\mathbf{3}$ 与环己酮和异丙醇

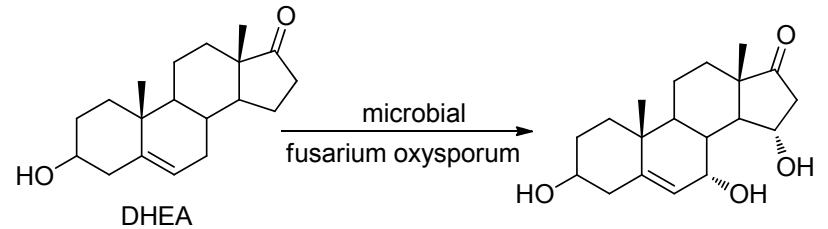

2
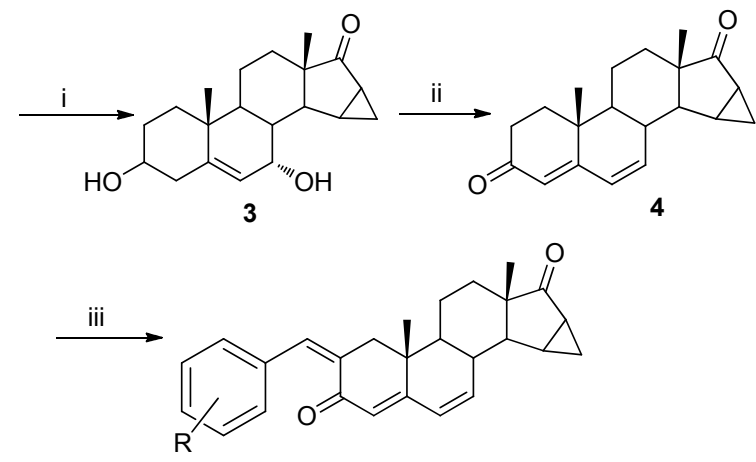

$\mathrm{R}=3-\mathrm{Cl}(\mathbf{5 a}), \mathrm{H}(\mathbf{5 b}), 2-\mathrm{F}(5 \mathbf{c}), 3-\mathrm{F}(\mathbf{5 d}), 2,4-\mathrm{F}_{2}(\mathbf{5 e})$, $2-\mathrm{Cl}(\mathbf{5 f}), 4-\mathrm{Br}(\mathbf{5 g}), 4-\mathrm{Cl}(\mathbf{5 h}), 3-\mathrm{OCH}_{3}(\mathbf{5 i})$, $3,4,5-(\mathrm{OMe})_{3}(5 \mathbf{j}), 2-\mathrm{OCH}_{3}(\mathbf{5 k})$

Reagents and conditions: (i) $\mathrm{Me}_{3} \mathrm{SOI}, \mathrm{NaOH}, \mathrm{DMSO}, 100{ }^{\circ} \mathrm{C}$, reflux, $50 \%$ yield; (ii) $\mathrm{Al}(\mathrm{O}-\mathrm{i}-\mathrm{Pr})_{3}$, cyclohexane, toluene, $100{ }^{\circ} \mathrm{C}$, reflux, $60 \%$ yield; (iii) substituted benzaldehyde, $\mathrm{NaOH}, \mathrm{EtOH}$, r.t., $80 \%$ $90 \%$ yield.

图式 1 目标化合物 $\mathbf{5 a} \sim \mathbf{5 k}$ 的合成路线

Scheme 1 Synthesis route of target compounds $\mathbf{5 a} \sim \mathbf{5 k}$

铝通过 Oppenauer 氧化得到 15 $\beta, 16 \beta$-亚甲基雄甾- 4,6-二 烯-3,17-二酮(4). 中间体 4 与各种取代苯甲醛通过克莱 森一施密特(Claisen-Schmidt)缩合反应得到系列衍生物 $\mathbf{5 a} \sim 5 \mathbf{k}$.

\section{2 抗炎活性}

首先建立体外 LPS 激活 BV2 小胶质细胞异常活化 的篮选模型，考察所合成的 11 个目标化合物对 LPS 激 活 BV2 小胶质细胞 $\mathrm{NO}$ 释放及细胞存活率的影响. 研究 结果如表 1 和表 2 所示, 可以看出所有目标化合物作用 $24 \mathrm{~h}$ 后对 LPS 活化的小鼠小胶质细胞株 BV2 的 NO 释 放都有不同程度的抑制作用，且具有一定的浓度依赖 性. 其中 $\mathbf{5 a}$ 和 $5 \mathbf{j}$ 的 $\mathrm{IC}_{50}$ 值分别为 2.69 和 $3.28 \mu \mathrm{mol} \cdot$ $\mathrm{L}^{-1}$, 有显著的抑制作用, 优于阳性对照米诺环素 $\left(\mathrm{IC}_{50}\right.$ 值为 $\left.5.97 \mu \mathrm{mol} \cdot \mathrm{L}^{-1}\right) .5 \mathbf{b} \sim 5 \mathbf{e}, 5 \mathbf{i}$ 也显示出了较好的抑制 活性, 其 $\mathrm{IC}_{50}$ 均 $<9.35 \mu \mathrm{mol} \cdot \mathrm{L}^{-1}$. 同时, $\mathbf{5 a}, \mathbf{5 c}$ 在浓度小 于 $10 \mu \mathrm{mol} \cdot \mathrm{L}^{-1}$ 下, $\mathbf{5 d}, \mathbf{5 e}, \mathbf{5 g}$ 在浓度在小于 $1 \mu \mathrm{mol} \cdot \mathrm{L}^{-1}$ 下不影响小胶质细胞的存活率.

\section{3 活性分析}

采用 Griess 法和四甲基偶氮唑盐微量酶反应比色法 (MTT) 分别考察了所合成化合物对 LPS 激活小胶质细胞 的抑制作用和对小胶质细存活率的影响. $5 \mathbf{a}$ 和 $5 \mathbf{j}$ 对 LPS 激活小胶质细胞的抑制作用最好, 而且优于米诺环素. 
表 1 化合物对 LPS 激活 BV2 小胶质细胞释放一氧化氮(\%)的影响(mean $\pm \mathrm{SE})^{a}$

Table 1 Effects of compounds on the release of nitric oxide (\%) by LPS-activated BV2 microglia(mean \pm SE)

\begin{tabular}{|c|c|c|c|c|c|c|c|}
\hline \multirow{2}{*}{ Compd. } & \multirow[b]{2}{*}{ Control } & \multirow{2}{*}{ LPS } & \multicolumn{4}{|c|}{ Comp. + LPS } & \multirow{2}{*}{$\mathrm{IC}_{50} /\left(\mu \mathrm{mol} \cdot \mathrm{L}^{-1}\right)$} \\
\hline & & & $1 \mu \mathrm{mol} \cdot \mathrm{L}^{-1}$ & $10 \mu \mathrm{mol} \cdot \mathrm{L}^{-1}$ & $30 \mu \mathrm{mol} \cdot \mathrm{L}^{-1}$ & $100 \mu \mathrm{mol} \cdot \mathrm{L}^{-1}$ & \\
\hline $5 a$ & $21.37 \pm 1.36$ & $100.00 \pm 2.45^{\# \# \#}$ & $91.08 \pm 1.50 * *$ & $26.76 \pm 0.95 * * *$ & $8.92 \pm 0.75 * * *$ & $6.22 \pm 0.72 * * *$ & 2.69 \\
\hline $5 \mathbf{b}$ & $21.37 \pm 1.36$ & $100.00 \pm 2.45^{\# \# \#}$ & $105.60 \pm 1.98$ & $49.17 \pm 1.65^{* *}$ & $5.39 \pm 0.55^{* *}$ & $5.39 \pm 0.21^{* *}$ & 8.50 \\
\hline $5 c$ & $21.37 \pm 1.36$ & $100.00 \pm 2.45^{\# \# \#}$ & $111.41 \pm 2.19^{* * *}$ & $38.59 \pm 1.57 * * *$ & $7.05 \pm 0.55^{* * *}$ & $6.85 \pm 0.95^{* * *}$ & 7.18 \\
\hline 5d & $21.37 \pm 1.36$ & $100.00 \pm 2.45^{\# \# \#}$ & $91.91 \pm 1.81^{* *}$ & $56.85 \pm 1.84 * * *$ & $9.34 \pm 0.72 * * *$ & $7.47 \pm 0.36^{* * *}$ & 6.11 \\
\hline $5 e$ & $16.14 \pm 1.49$ & $100.00 \pm 1.38^{\# \# \#}$ & $90.45 \pm 0.60^{* *}$ & $55.45 \pm 2.62 * * *$ & $25.45 \pm 1.38^{* * *}$ & $10.45 \pm 1.64 * * *$ & 6.91 \\
\hline $5 f$ & $16.14 \pm 1.49$ & $100.00 \pm 1.38^{\# \# \#}$ & $84.77 \pm 2.24 * * *$ & $84.55 \pm 1.42 * * *$ & $79.32 \pm 1.78 * * *$ & $39.55 \pm 0.39 * * *$ & 62.69 \\
\hline $5 \mathrm{~g}$ & $16.14 \pm 1.49$ & $100.00 \pm 1.38^{\# \# \#}$ & $85.68 \pm 3.53^{* *}$ & $74.55 \pm 2.90 * * *$ & $61.82 \pm 3.16^{* * *}$ & $31.82 \pm 1.20^{* * *}$ & 23.32 \\
\hline $5 \mathrm{~h}$ & $16.14 \pm 1.49$ & $100.00 \pm 1.38^{\# \# \#}$ & $90.45 \pm 2.19^{* *}$ & $70.91 \pm 1.04 * * *$ & $57.95 \pm 1.97 * * *$ & $20.91 \pm 1.98^{* * *}$ & 17.23 \\
\hline $5 \mathbf{i}$ & $16.14 \pm 1.49$ & $100.00 \pm 1.38^{\# \# \#}$ & $101.23 \pm 0.99$ & $52.95 \pm 1.14 * * *$ & $7.50 \pm 0.79 * * *$ & $7.05 \pm 0.45^{* * *}$ & 9.35 \\
\hline $5 \mathbf{j}$ & $16.14 \pm 1.49$ & $100.00 \pm 1.38^{\# \# \#}$ & $80.68 \pm 0.60^{* * *}$ & $42.95 \pm 1.97 * * *$ & $8.64 \pm 0.23^{* * *}$ & $7.50 \pm 0.39^{* * *}$ & 3.28 \\
\hline $5 k$ & $16.14 \pm 1.49$ & $100.00 \pm 1.38^{\# \# \#}$ & $110.91 \pm 1.20^{*}$ & $74.55 \pm 2.95^{*}$ & $18.86 \pm 0.60^{* * *}$ & $12.95 \pm 0.20^{* * *}$ & 12.71 \\
\hline MINO & $11.36 \pm 0.99$ & $100.00 \pm 0.99^{\# \# \#}$ & $96.74 \pm 2.71$ & $27.73 \pm 3.94 * * *$ & $18.64 \pm 2.98^{* * *}$ & $12.05 \pm 1.59 * * *$ & 5.97 \\
\hline
\end{tabular}

${ }^{a}$ Significance: ${ }^{*} p<0.05,{ }^{* *} p<0.01,{ }^{* * *} p<0.001$ compared to LPS group. ${ }^{* \# \#} p<0.001$ compared to control group. MINO: minocycline.

表 2 化合物对 LPS 活化的 BV2 小胶质细胞存活率(\%)的影响(mean $\pm \mathrm{SE})^{a}$

Table 2 Effect of compounds on the survival rate (\%) of LPS-activated BV2 microglia (mean \pm SE)

\begin{tabular}{|c|c|c|c|c|c|c|}
\hline \multirow{2}{*}{ Compd. } & \multirow{2}{*}{ Control } & \multirow{2}{*}{ LPS } & \multicolumn{4}{|c|}{ Comp. + LPS } \\
\hline & & & $1 \mu \mathrm{mol} \cdot \mathrm{L}^{-1}$ & $10 \mu \mathrm{mol} \cdot \mathrm{L}^{-1}$ & $30 \mu \mathrm{mol} \cdot \mathrm{L}^{-1}$ & $100 \mu \mathrm{mol} \cdot \mathrm{L}^{-1}$ \\
\hline $5 \mathbf{a}$ & $100.00 \pm 1.46$ & $92.92 \pm 1.38$ & $101.92 \pm 2.03$ & $94.39 \pm 2.37$ & $83.27 \pm 4.46^{* *}$ & $2.26 \pm 0.08 * * *$ \\
\hline $5 c$ & $100.00 \pm 1.46$ & $92.92 \pm 1.38$ & $84.97 \pm 2.85$ & $80.44 \pm 4.16$ & $17.69 \pm 1.10 * *$ & $6.16 \pm 0.31 * * *$ \\
\hline $5 d$ & $100.00 \pm 1.46$ & $92.92 \pm 1.38$ & $83.14 \pm 3.88$ & $68.54 \pm 1.38^{*}$ & $63.45 \pm 3.90 * *$ & $2.24 \pm 0.14 * *$ \\
\hline $5 e$ & $100.00 \pm 0.49$ & $95.41 \pm 2.27$ & $99.21 \pm 1.01$ & $91.09 \pm 3.38^{*}$ & $83.03 \pm 4.05 * *$ & $76.05 \pm 2.80 * * *$ \\
\hline $5 f$ & $100.00 \pm 0.49$ & $95.41 \pm 2.27$ & $84.77 \pm 3.4^{* *}$ & $77.16 \pm 4.29 * * *$ & $73.97 \pm 0.8 * * *$ & $71.86 \pm 4.2 * * *$ \\
\hline $5 \mathrm{~g}$ & $100.00 \pm 0.49$ & $95.41 \pm 2.27$ & $90.80 \pm 2.34$ & $88.75 \pm 3.62 *$ & $87.14 \pm 4.73^{*}$ & $79.20 \pm 4.64 * *$ \\
\hline $5 h$ & $100.00 \pm 0.49$ & $95.41 \pm 2.27$ & $90.64 \pm 2.94 *$ & $90.05 \pm 2.98 *$ & $80.01 \pm 1.28 * *$ & $79.15 \pm 1.90 * *$ \\
\hline $5 \mathbf{i}$ & $100.00 \pm 0.49$ & $95.41 \pm 2.27$ & $71.07 \pm 2.56^{*}$ & $65.69 \pm 2.15^{*}$ & $9.72 \pm 2.26^{*}$ & $4.81 \pm 0.08 * * *$ \\
\hline $5 \mathbf{j}$ & $100.00 \pm 0.49$ & $95.41 \pm 2.27$ & $72.03 \pm 4.23 * * *$ & $71.00 \pm 3.34 * * *$ & $69.33 \pm 4.91^{* * *}$ & $37.80 \pm 4.80 * * *$ \\
\hline $5 k$ & $100.00 \pm 0.49$ & $95.41 \pm 2.27$ & $82.30 \pm 4.13 * *$ & $67.08 \pm 4.39 * * *$ & $61.86 \pm 4.52 * * *$ & $26.63 \pm 3.09 * * *$ \\
\hline MINO & $100.00 \pm 0.11$ & $94.20 \pm 1.86$ & $98.54 \pm 1.83$ & $89.65 \pm 3.02$ & $88.50 \pm 4.44$ & $83.49 \pm 3.22 *$ \\
\hline
\end{tabular}

${ }^{a}$ Significance: ${ }^{*} p<0.05,{ }^{* *} p<0.01,{ }^{* * *} p<0.001$ compared to control group.

对目标化合物进行构效(SAR)分析，苯环间位取代物的 $\mathrm{IC}_{50}$ 值均较邻位取代物的 $\mathrm{IC}_{50}$ 值小, 说明间位取代修饰 更有利于提高化合物的抗炎活性. 将化合物 $5 \mathbf{i} \sim 5 \mathbf{k}$ 的 $\mathrm{IC}_{50}$ 值进行比较, 3,4,5-三甲氧基取代的 $\mathbf{5} \mathbf{j}$ 虽然取代基团 较大, 但却表现出很好的抗炎活性, 可能与取代基的空 间位置和与靶点的相互作用有关, 通过分析可以得出间 位取代的 2-去氢表雄酮苯亚甲基化合物具有较好的抗 炎活性, 可作为治疗 $\mathrm{AD}$ 的先导化合物进一步修饰研究.

\section{2 结论}

合成了 11 个 $15 \beta, 16 \beta$-亚甲基雄甾-2-取代苯亚甲基4,6-二烯-3,17-二酮全新衍生物. 通过微生物发酵的方 法, 在 DHEA 中的 7、15 位上引入了两个羟基, 然后制 备中间体 3, 大大缩短了化学合成步骤, 属于比较新颖 的方法. 且由化合物 $\mathbf{2}$ 一步温和、高效地合成中间体 $\mathbf{3}$ 未有文献报道, 具有创新性. 经文献调研, 主要是先将 中间体 2 的 3 位和 15 位羟基用醋酸酐或特戊酰氯保护,
然后再与硫叶立德试剂反应, 生成中间体 $\mathbf{3}$, 但本课题 组前期研究发现 15 位羟基保护的化合物和未保护的化 合物在相同条件下得到了相同物质中间体 $\mathbf{3}$, 为一步合 成三元环碳提供了新方法.

去氢表雄酮及其硫酸酯(DHEA-S)作为神经甾体类 物质, 被证明与短期记忆和长期记忆都有关系. 但目前 还没有 DHEA 用于 AD 治疗的明确报道, 我们在其结构 中引入三元碳环和查尔酮结构, 可能由于两者结构的同 源性和查尔酮结构单元的良好抗炎活性，在对抑制小胶 质细胞激活为靶点的研究中表现出了较好的抑制活性, 说明将这两个结构拼合在一起的改造是成功的, 但具体 的作用机制还有待于进一步的深入研究.

\section{3 实验部分}

\section{1 仪器与试剂}

${ }^{1} \mathrm{H}$ NMR 和 ${ }^{13} \mathrm{C}$ NMR 谱使用瑞典 Bruker 公司 AVANCE III-400 型超导核磁共振仪测定, TMS 为内标; 
高分辨质谱使用美国 Waters-Micromass 公司 Q-Tof Micro 高分辨四级杆-飞行时间串联质谱仪测定; 硅胶: 中 国青岛海洋化工集团公司, 柱色谱硅胶: 上海五四化学 试剂厂. 三甲基碘化亚砜、异丙醇铝、各种取代苯甲醛 均购于阿拉丁试剂公司, 去氢表雄酮购于汉中汉江振华 生物科技有限公司. 本行业有机溶剂的分离纯化均为工 业级蒸馏再使用, 其他试剂均为市售分析试剂.

\section{2 化合物的合成}

\subsubsection{3 $3 \beta, 7 \alpha, 15 \alpha$-三差基-5-烯-雄留-17-酮(2)的合成}

配制培养基: 麦芽糖、蛋白炼、酵母膏和磷酸氢二 钠质量分数分别为 $2.65 \%, 1.45 \%, 0.02 \%$ 和 $0.1 \%, \mathrm{pH}$ 为 5 , 每瓶装液量为 $100 \mathrm{~mL} / 500 \mathrm{~mL}$, 转化温度 $30{ }^{\circ} \mathrm{C}$, 培 养 $48 \mathrm{~h}$ 后, 将底物去氢表雄酮用丙酮溶解加入转化液, 底物终浓度为 $1 \mathrm{~g} / \mathrm{L}$, 转化 $96 \mathrm{~h}$. 将菌丝体与培养液抽滤 分离, 培养滤液用乙酸乙酯萃取三遍, 菌丝体用适量乙 酸乙酯浸泡后超声震荡三次(每次大于 $10 \mathrm{~min}$ )后, 合并 乙酸乙酯萃取液, 将萃取液进行减压蒸馏. 浓缩至 200 $\mathrm{mL}$ 之后, 分别用饱和碳酸氢钠、水与饱和食盐水各洗 涤三遍以去除浓缩液中的杂质, 最后用无水硫酸镁干燥 后过滤, 将滤液进行减压蒸馏, 得到淡黄色固体, 转化 产物粗品经柱层析 $[V($ 氯仿 $): V$ (甲醇 $)=20: 1]$ 分离, 得 到化合物 2, 白色固体, m.p. $215.3 \sim 216.7{ }^{\circ} \mathrm{C}$, 具体转化 方法及优化条件等细节见文献 $[9,10]$.

3.2.2 $15 \beta, 16 \beta$-亚甲基- $3 \beta, 7 \alpha$-二羟基-5-烯-雄甾-17酮(3)的合成

将三甲基碘化亚砜(31.25 mmol)和 $2.5 \mathrm{~g}$ (62.5 $\mathrm{mmol}$ 氢氧化钠投入到 $100 \mathrm{~mL}$ 圆底烧瓶中加入 $50 \mathrm{~mL}$ 二甲基亚砜(DMSO)溶解, 室温下搅拌 $0.5 \mathrm{~h}$, 然后加入 $10 \mathrm{~g}$ (31.25 mmol) 化合物 2, 室温搅拌 $0.5 \mathrm{~h}$, 然后加热至 $100{ }^{\circ} \mathrm{C}$ 反应摚拌 $1 \mathrm{~h}$, 薄层色谱(TLC)监测, 至反应结束 后将溶液缓慢加入 $300 \mathrm{~mL}$ 饱和食盐水中, 析出棕色固 体, 过滤后得化合物 3 粗品, 之后采用柱色谱分离纯化 $[V($ 石油醚 $): V($ 乙酸乙酯 $)=1: 2]$, 得到中间体 $\mathbf{3}^{[10]}$. 白 色固体, 产率 50\%. m.p. 186.1 $187.3{ }^{\circ} \mathrm{C} ;{ }^{1} \mathrm{H}$ NMR (400 $\left.\mathrm{MHz}, \mathrm{CDCl}_{3}\right) \delta: 5.48(\mathrm{~d}, J=4.4 \mathrm{~Hz}, 1 \mathrm{H}), 4.68(\mathrm{~d}, J=4.0$ $\mathrm{Hz}, 1 \mathrm{H}), 4.18(\mathrm{~d}, J=8.0 \mathrm{~Hz}, 1 \mathrm{H}), 3.91 \sim 3.96(\mathrm{~m}, 1 \mathrm{H})$, $3.30 \sim 3.32(\mathrm{~m}, 1 \mathrm{H}), 2.09 \sim 2.22(\mathrm{~m}, 4 \mathrm{H}), 1.64 \sim 1.71(\mathrm{~m}$, $3 \mathrm{H}), 1.59 \sim 1.62(\mathrm{~m}, 1 \mathrm{H}), 1.55 \sim 1.60(\mathrm{~m}, 2 \mathrm{H}), 1.51 \sim 1.54$ $(\mathrm{m}, 1 \mathrm{H}), 1.41 \sim 1.43(\mathrm{~m}, 2 \mathrm{H}), 1.34 \sim 1.37(\mathrm{~m}, 1 \mathrm{H}), 1.12 \sim$ $1.16(\mathrm{~m}, 2 \mathrm{H}), 0.99 \sim 1.06(\mathrm{~m}, 1 \mathrm{H}), 0.92(\mathrm{~s}, 3 \mathrm{H}), 0.86(\mathrm{~s}$, $3 \mathrm{H}) ;{ }^{13} \mathrm{C} \mathrm{NMR}\left(100 \mathrm{MHz}, \mathrm{CDCl}_{3}\right) \delta: 216.91,146.98$, $123.57,71.16,64.98,46.43,42.99,41.96,37.74,36.80$, $36.01,35.12,31.26,25.84,22.49,19.82,19.66,18.03$, 17.28 .
3.2.3 15 $\beta, 16 \beta$-亚甲基雄甾-4,6-二烯-3,17-二酮(4)的 合成

取 $5 \mathrm{~g}(15.8 \mathrm{mmol})$ 化合物 3 于 $250 \mathrm{~mL}$ 圆底烧瓶中, 加入 $100 \mathrm{~mL}$ 甲苯加热溶解后加入 $6.538 \mathrm{~mL}(64 \mathrm{mmol})$ 环已酮, 搭分水装置 $120{ }^{\circ} \mathrm{C}$ 下回流除水, 待蒸出的甲苯 澄清后降温加入 $1.617 \mathrm{~g}(7.9 \mathrm{mmol})$ 异丙醇铝, 加热至 $100{ }^{\circ} \mathrm{C}$ 回流摚拌反应, TLC 检测反应完全后, 溶液先用 质量分数为 $10 \%$ 的硫酸洗 3 遍，再用饱和碳酸氢钠洗一 次，最后用饱和食盐水洗 3 次. 然后加入无水硫酸镁干 燥过夜, 减压蒸馏除去甲苯, 得黄色油状物质, 经柱层 析 $[V$ (石油醚) $: V($ 乙酸乙酯 $)=5 ： 1]$ 分离得中间体 $4^{[11]}$. 白色固体，产率 $60 \%$. m.p. 150.9 $152.0{ }^{\circ} \mathrm{C} ;{ }^{1} \mathrm{H}$ NMR $\left(400 \mathrm{MHz}, \mathrm{CDCl}_{3}\right) \delta: 6.39(\mathrm{dd}, J=9.7,1.8 \mathrm{~Hz}, 1 \mathrm{H}), 6.24$ $(\mathrm{dd}, J=9.8,2.7 \mathrm{~Hz}, 1 \mathrm{H}), 5.72(\mathrm{~s}, 1 \mathrm{H}), 2.56$ (ddd, $J=18.2$, 14.3, $5.4 \mathrm{~Hz}, 1 \mathrm{H}), 2.47$ (d, $J=2.0 \mathrm{~Hz}, 1 \mathrm{H}), 2.14$ (ddd, $J=$ 14.1, 8.3, $3.9 \mathrm{~Hz}, 2 \mathrm{H}$ ), 1.99 (ddd, $J=13.1,5.4,2.1 \mathrm{~Hz}$, $1 \mathrm{H}), 1.83(\mathrm{dd}, J=5.7,3.0 \mathrm{~Hz}, 2 \mathrm{H}), 1.78(\mathrm{~d}, J=4.0,1 \mathrm{H})$, $1.71 \sim 1.75(\mathrm{~m}, 2 \mathrm{H}), 1.68 \sim 1.70(\mathrm{~m}, 1 \mathrm{H}), 1.41 \sim 1.45(\mathrm{~m}$, $1 \mathrm{H}), 1.34 \sim 1.38(\mathrm{~m}, 2 \mathrm{H}), 1.23 \sim 1.28(\mathrm{~m}, 1 \mathrm{H}), 1.15(\mathrm{~s}$, $3 \mathrm{H}), 1.07(\mathrm{~s}, 3 \mathrm{H}) ;{ }^{13} \mathrm{C} \mathrm{NMR}\left(100 \mathrm{MHz}, \mathrm{CDCl}_{3}\right) \delta: 215.21$, $199.29,163.08,138.61,128.99,124.23,51.53,49.75$, $42.99,36.27,35.93,35.06,33.86,33.61,25.95,21.61$, $20.23,20.02,17.43,16.19$.

\section{2 .4 化合物 $\mathbf{5 a} \sim \mathbf{5 k}$ 的合成}

取 $150 \mathrm{mg}$ (0.5 mmol) 化合物 4 于 $25 \mathrm{~mL}$ 圆底烧瓶中, 加入 $10 \mathrm{~mL}$ 无水乙醇溶解后加入 $40 \mathrm{mg}(1.0 \mathrm{mmol})$ $\mathrm{NaOH}$ 室温下搅拌 $30 \mathrm{~min}$, 再加入不同取代的苯甲醛(1 equiv.)室温条件下反应, TLC 跟踪监测. 反应结束后, 减 压蒸馏除去乙醇, 加入乙酸乙酯溶解后分别用水和饱和 食盐水萃取 3 次, 加入无水硫酸镁干燥过夜后采用柱色 谱分离纯化化合物 $[V$ (石油醚) : $V$ (乙酸乙酯 $)=3: 1]$, 得目标化合物 $\mathbf{5 a} \sim \mathbf{5 k}$.

15 $\beta, 16 \beta$-亚甲基雄甾-2-(3-氯)苯亚甲基-4,6-二烯3,17-二酮 (5a): 黄色固体，产率 80\%. m.p. 183.9 $185.0{ }^{\circ} \mathrm{C} ;{ }^{1} \mathrm{H}$ NMR (400 MHz, $\left.\mathrm{CDCl}_{3}\right) \delta: 7.64$ (d, $J=2.7$ $\mathrm{Hz}, 1 \mathrm{H}), 7.38 \sim 7.27(\mathrm{~m}, 3 \mathrm{H}), 7.23(\mathrm{dt}, J=6.6,1.7 \mathrm{~Hz}$, $1 \mathrm{H}), 6.44(\mathrm{dd}, J=9.6,2.0 \mathrm{~Hz}, 1 \mathrm{H}), 6.32(\mathrm{dd}, J=9.8,2.7$ $\mathrm{Hz}, 1 \mathrm{H}), 5.95(\mathrm{~s}, 1 \mathrm{H}), 2.48 \sim 2.37(\mathrm{~m}, 2 \mathrm{H}), 2.20 \sim 2.09(\mathrm{~m}$, $2 \mathrm{H}), 1.87 \sim 1.65(\mathrm{~m}, 5 \mathrm{H}), 1.49 \sim 1.34(\mathrm{~m}, 3 \mathrm{H}), 1.23(\mathrm{~d}, J=$ $6.2 \mathrm{~Hz}, 1 \mathrm{H}), 1.03(\mathrm{~s}, 3 \mathrm{H}), 1.01(\mathrm{~s}, 3 \mathrm{H}) ;{ }^{13} \mathrm{C} \mathrm{NMR}(101$ $\left.\mathrm{MHz}, \mathrm{CDCl}_{3}\right) \delta: 214.96,188.19,162.08,139.04,137.64$, $134.68,134.49$, 134.43, 129.76, 129.40, 128.65, 128.31, $127.49,124.81,50.78,49.75,42.92,38.04,37.93,35.80$, 35.03, 25.98, 21.54, 20.19, 20.15, 18.56, 17.39; HRMS (ESI) calcd for $\mathrm{C}_{27} \mathrm{H}_{28} \mathrm{ClO}_{2}[\mathrm{M}+\mathrm{H}]^{+}$419.1778, found 
419.1779 .

15 $\beta, 16 \beta$-亚甲基雄甾-2-苯亚甲基-4,6-二烯-3,17-二 酩(5b): 淡黄色固体, 产率 91\%. m.p. $116.3 \sim 118.1{ }^{\circ} \mathrm{C}$; ${ }^{1} \mathrm{H}$ NMR $\left(400 \mathrm{MHz}, \mathrm{CDCl}_{3}\right) \delta: 7.66(\mathrm{~d}, J=2.8 \mathrm{~Hz}, 1 \mathrm{H})$, $7.37 \sim 7.24(\mathrm{~m}, 5 \mathrm{H}), 6.35(\mathrm{dd}, J=9.7,2.1 \mathrm{~Hz}, 1 \mathrm{H}), 6.24$ (dd, $J=9.7,2.7 \mathrm{~Hz}, 1 \mathrm{H}), 5.88(\mathrm{~s}, 1 \mathrm{H}), 2.41 \sim 2.30(\mathrm{~m}, 2 \mathrm{H})$, $2.14 \sim 2.02(\mathrm{~m}, 2 \mathrm{H}), 1.75$ (ddd, $J=12.7,6.1,3.4 \mathrm{~Hz}, 2 \mathrm{H})$, $1.67 \sim 1.60(\mathrm{~m}, 2 \mathrm{H}), 1.42 \sim 1.28(\mathrm{~m}, 3 \mathrm{H}), 1.22 \sim 1.16(\mathrm{~m}$, 2H), 0.95 (s, 3H), 0.93 (s, 3H); ${ }^{13} \mathrm{C}$ NMR (101 MHz, $\left.\mathrm{CDCl}_{3}\right) \delta: 214.15,187.61,160.80,137.76,135.38,134.75$, $132.18,128.59,127.68,127.46,127.32,123.91,49.78$, 48.73, 41.91, 37.06, 36.82, 34.73, 34.01, 24.97, 20.58, $19.18,19.13,17.49,16.41$; HRMS (ESI) calcd for $\mathrm{C}_{27} \mathrm{H}_{29} \mathrm{O}_{2}[\mathrm{M}+\mathrm{H}]^{+}$385.2167, found 385.2166.

15 $\beta, 16 \beta$-亚甲基雄甾-2-(2-氟)-苯亚甲基-4,6-二烯3,17-二酮 $(\mathbf{5 c})$ : 淡黄色固体, 产率 83\%. m.p. 196.7 $198.3{ }^{\circ} \mathrm{C} ;{ }^{1} \mathrm{H}$ NMR $\left(400 \mathrm{MHz}, \mathrm{CDCl}_{3}\right) \delta: 7.73 \sim 7.66(\mathrm{~m}$, 1H), 7.32 (dtd, $J=13.6,8.3,7.8,1.8 \mathrm{~Hz}, 2 \mathrm{H}), 7.21 \sim 7.08$ (m, 2H), 6.43 (dd, $J=9.6,2.0 \mathrm{~Hz}, 1 \mathrm{H}), 6.32$ (dd, $J=9.7$, $2.7 \mathrm{~Hz}, 1 \mathrm{H}), 5.96(\mathrm{~s}, 1 \mathrm{H}), 2.42(\mathrm{td}, J=13.2,12.0,5.9 \mathrm{~Hz}$, $2 \mathrm{H}), 2.20 \sim 2.08(\mathrm{~m}, 2 \mathrm{H}), 1.86 \sim 1.62(\mathrm{~m}, 5 \mathrm{H}), 1.40$ (ddd, $J=21.9,6.8,3.0 \mathrm{~Hz}, 3 \mathrm{H}), 1.27 \sim 1.19(\mathrm{~m}, 1 \mathrm{H}), 1.01(\mathrm{~s}$, $3 \mathrm{H}), 1.00(\mathrm{~s}, 3 \mathrm{H}) ;{ }^{13} \mathrm{C} \mathrm{NMR}\left(101 \mathrm{MHz}, \mathrm{CDCl}_{3}\right) \delta: 215.09$, 188.06, 162.24, 138.91, 135.44, $130.51(\mathrm{~d}, J=3.1 \mathrm{~Hz})$, $130.21,130.13,129.37$ (d, $J=2.4 \mathrm{~Hz}), 128.72,124.85$, 123.91 (d, $J=3.6 \mathrm{~Hz}), 116.00,115.78,50.81,49.75,42.94$, $38.51,37.92,35.77,35.02,25.98,21.57,20.16,20.15$, 18.38, 17.40; HRMS (ESI) calcd for $\mathrm{C}_{27} \mathrm{H}_{28} \mathrm{FO}_{2}[\mathrm{M}+\mathrm{H}]^{+}$ 403.2073, found 403.2072.

15 $\beta, 16 \beta$-亚甲基雄甾-2-(3-氟)-苯亚甲基-4,6-二烯3,17-二酮 (5d): 淡黄色固体, 产率 80\%. m.p. 130.1 $131.7{ }^{\circ} \mathrm{C} ;{ }^{1} \mathrm{H}$ NMR $\left(400 \mathrm{MHz}, \mathrm{CDCl}_{3}\right) \delta: 7.67$ (d, $J=2.8$ $\mathrm{Hz}, 1 \mathrm{H}), 7.38$ (td, $J=7.8,5.9 \mathrm{~Hz}, 1 \mathrm{H}), 7.13(\mathrm{~d}, J=7.6 \mathrm{~Hz}$, $1 \mathrm{H}), 7.08 \sim 7.00(\mathrm{~m}, 2 \mathrm{H}), 6.44(\mathrm{dd}, J=9.6,2.0 \mathrm{~Hz}, 1 \mathrm{H})$, $6.32(\mathrm{dd}, J=9.7,2.7 \mathrm{~Hz}, 1 \mathrm{H}), 5.96(\mathrm{~s}, 1 \mathrm{H}), 2.47 \sim 2.38(\mathrm{~m}$, 2H), $2.20 \sim 2.09$ (m, 2H), 1.83 (ddd, $J=10.3,4.9,2.1 \mathrm{~Hz}$, $2 \mathrm{H}), 1.70(\mathrm{dtd}, J=8.7,5.8,2.5 \mathrm{~Hz}, 2 \mathrm{H}), 1.48 \sim 1.43(\mathrm{~m}$, $2 \mathrm{H}), 1.26(\mathrm{t}, J=7.1 \mathrm{~Hz}, 3 \mathrm{H}), 1.03$ (s, 3H), $1.01(\mathrm{~s}, 3 \mathrm{H}) ;{ }^{13} \mathrm{C}$ NMR $\left(101 \mathrm{MHz}, \mathrm{CDCl}_{3}\right) \delta$ : 215.07, 188.30, 162.09, 139.03, 134.93 (d, $J=2.4 \mathrm{~Hz}), 134.30,130.06$ (d, $J=8.4$ $\mathrm{Hz}), 128.66,125.31$ (d, $J=2.8 \mathrm{~Hz}), 124.81,116.24$, $116.03,115.34,115.13,50.76,49.73,42.93,38.04,37.91$, $35.78,35.01,25.99,21.57,20.21,20.15,18.56,17.42$; HRMS (ESI) calcd for $\mathrm{C}_{27} \mathrm{H}_{28} \mathrm{FO}_{2}[\mathrm{M}+\mathrm{H}]^{+}$403.2073, found 403.2072 .
15 $\beta, 16 \beta$-亚甲基雄甾-2-(2,4-二氟)-苯亚甲基-4,6-二 烯-3,17-二酮(5e): 黄色固体, 产率 90\%. m.p. 201.2 $203.9{ }^{\circ} \mathrm{C} ;{ }^{1} \mathrm{H}$ NMR $\left(400 \mathrm{MHz}, \mathrm{CDCl}_{3}\right) \delta: 7.64 \sim 7.58(\mathrm{~m}$, $1 \mathrm{H}), 7.30 \sim 7.21(\mathrm{~m}, 1 \mathrm{H}), 6.96 \sim 6.84(\mathrm{~m}, 2 \mathrm{H}), 6.43(\mathrm{dd}$, $J=9.7,2.0 \mathrm{~Hz}, 1 \mathrm{H}), 6.32(\mathrm{dd}, J=9.7,2.7 \mathrm{~Hz}, 1 \mathrm{H}), 5.95(\mathrm{~s}$, $1 \mathrm{H}), 2.41(\mathrm{td}, J=12.7,11.3,3.0 \mathrm{~Hz}, 2 \mathrm{H}), 2.20 \sim 2.08(\mathrm{~m}$, $2 \mathrm{H}), 1.86 \sim 1.62(\mathrm{~m}, 5 \mathrm{H}), 1.49 \sim 1.31(\mathrm{~m}, 3 \mathrm{H}), 1.28 \sim 1.19$ (m, 1H), 1.02 (s, 3H), 1.00 (s, 3H); ${ }^{13} \mathrm{C}$ NMR $(101 \mathrm{MHz}$, $\left.\mathrm{CDCl}_{3}\right) \delta: 215.01,187.87,162.23,139.00,135.51,131.32$ (dd, $J=9.6,4.6 \mathrm{~Hz}$ ), 128.6, 124.78, 119.89 (dd, $J=14.9$, $3.9 \mathrm{~Hz}), 111.50(\mathrm{~d}, J=3.7 \mathrm{~Hz}), 111.28(\mathrm{~d}, J=3.6 \mathrm{~Hz})$, 104.67, 104.42, 104.17, 50.79, 49.74, 42.93, 38.52 (d, $J=$ $2.0 \mathrm{~Hz}), 37.92,35.79,35.01,25.98,21.56,20.19,20.14$, 18.41, 17.40; HRMS (ESI) calcd for $\mathrm{C}_{27} \mathrm{H}_{27} \mathrm{~F}_{2} \mathrm{O}_{2}[\mathrm{M}+\mathrm{H}]^{+}$ 421.1978, found 421.1978 .

15 $\beta, 16 \beta$-亚甲基雄甾-2-(2-氯)-苯亚甲基-4,6-二烯3,17-二酮 (5f): 黄色固体, 产率 85\%. m.p. 236.1 $237.2{ }^{\circ} \mathrm{C} ;{ }^{1} \mathrm{H}$ NMR $\left(400 \mathrm{MHz}, \mathrm{CDCl}_{3}\right) \delta: 7.74(\mathrm{~d}, J=2.7$ $\mathrm{Hz}, 1 \mathrm{H}), 7.47 \sim 7.41(\mathrm{~m}, 1 \mathrm{H}), 7.31 \sim 7.27(\mathrm{~m}, 2 \mathrm{H}), 7.25 \sim$ $7.20(\mathrm{~m}, 1 \mathrm{H}), 6.42$ (dd, $J=9.7,2.0 \mathrm{~Hz}, 1 \mathrm{H}), 6.32$ (dd, $J=$ 9.8, $2.7 \mathrm{~Hz}, 1 \mathrm{H}), 5.96(\mathrm{~s}, 1 \mathrm{H}), 2.43 \sim 2.35(\mathrm{~m}, 2 \mathrm{H}), 2.19 \sim$ $2.07(\mathrm{~m}, 2 \mathrm{H}), 1.86 \sim 1.65(\mathrm{~m}, 4 \mathrm{H}), 1.59(\mathrm{dd}, J=8.8,3.4$ $\mathrm{Hz}, 1 \mathrm{H}), 1.39 \sim 1.17(\mathrm{~m}, 4 \mathrm{H}), 1.00(\mathrm{~s}, 6 \mathrm{H}) ;{ }^{13} \mathrm{C}$ NMR $(101$ $\left.\mathrm{MHz}, \mathrm{CDCl}_{3}\right) \delta: 215.12,188.39,162.24,138.95,134.95$, $134.66,134.48,133.56,130.09,129.74,129.43,128.70$, $126.41,124.88,50.80,49.72,42.92,38.21,38.01,35.78$, $34.97,26.08 \sim 25.88(\mathrm{~m}), 21.58,20.13,18.19,17.42$; HRMS (ESI) calcd for $\mathrm{C}_{27} \mathrm{H}_{28} \mathrm{ClO}_{2}[\mathrm{M}+\mathrm{H}]^{+} 419.1778$, found 419.1777 .

15 $\beta$,16 $\beta$-亚甲基雄甾-2-(4-溴)-苯亚甲基-4,6-二烯3,17-二酮 (5g): 黄色固体, 产率 75\%. m.p. 221.1 $222.3{ }^{\circ} \mathrm{C} ;{ }^{1} \mathrm{H}$ NMR $\left(400 \mathrm{MHz}, \mathrm{CDCl}_{3}\right) \delta: 7.64(\mathrm{~d}, J=2.7$ $\mathrm{Hz}, 1 \mathrm{H}), 7.56 \sim 7.50(\mathrm{~m}, 2 \mathrm{H}), 7.26 \sim 7.20(\mathrm{~m}, 2 \mathrm{H}), 6.43$ (dd, $J=9.7,2.0 \mathrm{~Hz}, 1 \mathrm{H}), 6.31(\mathrm{dd}, J=9.7,2.7 \mathrm{~Hz}, 1 \mathrm{H})$, $5.95(\mathrm{~s}, 1 \mathrm{H}), 2.47 \sim 2.37(\mathrm{~m}, 2 \mathrm{H}), 2.20 \sim 2.08(\mathrm{~m}, 2 \mathrm{H})$, $1.87 \sim 1.65(\mathrm{~m}, 5 \mathrm{H}), 1.48 \sim 1.33(\mathrm{~m}, 3 \mathrm{H}), 1.24(\mathrm{qd}, J=8.3$, 7.7, $5.6 \mathrm{~Hz}, 1 \mathrm{H}), 1.03$ (s, 3H), 0.99 (s, 3H); ${ }^{13} \mathrm{C}$ NMR (101 $\left.\mathrm{MHz}, \mathrm{CDCl}_{3}\right) \delta: 215.05,188.28,161.92,138.96,135.00$, $134.65,133.92,131.71,131.07,128.64,124.82,122.49$, $50.75,49.71,42.91,38.09,37.87,35.76,34.99,25.98$, 21.56, 20.23, 20.14, 18.54, 17.41; HRMS (ESI) calcd for $\mathrm{C}_{27} \mathrm{H}_{28} \mathrm{BrO}_{2}[\mathrm{M}+\mathrm{H}]^{+}$463.1272, found 463.1271.

15 $\beta, 16 \beta$-亚甲基雄甾-2-(4-氯)-苯亚甲基-4,6-二烯3,17-二酮 (5h): 黄色固体, 产率 84\%. m.p. 212.8 $214.1{ }^{\circ} \mathrm{C} ;{ }^{1} \mathrm{H}$ NMR $\left(400 \mathrm{MHz}, \mathrm{CDCl}_{3}\right) \delta: 7.66(\mathrm{~d}, J=2.7$ 
$\mathrm{Hz}, 1 \mathrm{H}), 7.41 \sim 7.34(\mathrm{~m}, 2 \mathrm{H}), 7.32 \sim 7.26(\mathrm{~m}, 2 \mathrm{H}), 6.43$ (dd, $J=9.6,2.1 \mathrm{~Hz}, 1 \mathrm{H}), 6.31$ (dd, $J=9.8,2.7 \mathrm{~Hz}, 1 \mathrm{H})$, $5.95(\mathrm{~s}, 1 \mathrm{H}), 2.47 \sim 2.36(\mathrm{~m}, 2 \mathrm{H}), 2.21 \sim 2.08(\mathrm{~m}, 2 \mathrm{H})$, $1.88 \sim 1.66(\mathrm{~m}, 5 \mathrm{H}), 1.49 \sim 1.36(\mathrm{~m}, 3 \mathrm{H}), 1.27 \sim 1.21(\mathrm{~m}$, 1H), $1.02(\mathrm{~s}, 3 \mathrm{H}), 0.99(\mathrm{~s}, 3 \mathrm{H}) ;{ }^{13} \mathrm{C} \mathrm{NMR}(101 \mathrm{MHz}$, $\left.\mathrm{CDCl}_{3}\right) \delta: 215.07,188.32,161.97,138.99,135.01,134.24$, $134.20,133.83,130.83,128.76,128.64,124.80,50.75$, $49.72,42.91,38.08,37.87,35.76,35.00,25.98,21.58$, 20.23, 20.14, 18.54, 17.42; HRMS (ESI) calcd for $\mathrm{C}_{27} \mathrm{H}_{28^{-}}$ $\mathrm{ClO}_{2}[\mathrm{M}+\mathrm{H}]^{+}$419.1778, found 419.1776.

$15 \beta, 16 \beta$-亚甲基雄甾-2-(3-甲氧基)-苯亚甲基-4,6-二 烯-3,17-二酮(5i)：黄色固体，产率 79\%. m.p. 124.9 $126.4{ }^{\circ} \mathrm{C} ;{ }^{1} \mathrm{H}$ NMR (400 MHz, $\left.\mathrm{CDCl}_{3}\right) \delta 7.69$ (d, $J=2.7$ $\mathrm{Hz}, 1 \mathrm{H}), 7.32(\mathrm{td}, J=7.5,1.5 \mathrm{~Hz}, 1 \mathrm{H}), 6.98 \sim 6.94(\mathrm{~m}$, $1 \mathrm{H}), 6.88(\mathrm{dd}, J=8.5,1.9 \mathrm{~Hz}, 2 \mathrm{H}), 6.42(\mathrm{dd}, J=9.6,2.0$ $\mathrm{Hz}, 1 \mathrm{H}), 6.31$ (dd, $J=9.8,2.7 \mathrm{~Hz}, 1 \mathrm{H}), 5.95$ (s, 1H), 3.82 (s, 3H), $2.42(\mathrm{dt}, J=19.8,6.7 \mathrm{~Hz}, 2 \mathrm{H}), 2.20 \sim 2.09(\mathrm{~m}$, 2H), 1.83 (ddd, $J=12.6,6.0,3.3 \mathrm{~Hz}, 2 \mathrm{H}), 1.73 \sim 1.67(\mathrm{~m}$, $1 \mathrm{H}), 1.51 \sim 1.33(\mathrm{~m}, 3 \mathrm{H}), 1.26(\mathrm{t}, J=7.2 \mathrm{~Hz}, 2 \mathrm{H}), 1.02(\mathrm{~d}$, $J=5.0 \mathrm{~Hz}, 6 \mathrm{H}) ;{ }^{13} \mathrm{C}$ NMR $\left(101 \mathrm{MHz}, \mathrm{CDCl}_{3}\right) \delta: 215.13$, $188.58,161.89$, 159.52, 138.81, 137.13, 136.26, 133.46, $129.47,128.70,124.92,121.88,115.19,113.83,55.27$, $50.79,49.74,42.92,38.12,37.85,35.75,35.04,25.99$, 21.59, 20.21, 20.15, 18.52, 17.42; HRMS (ESI) calcd for $\mathrm{C}_{28} \mathrm{H}_{31} \mathrm{O}_{3}[\mathrm{M}+\mathrm{H}]^{+}$415.2773, found 415.2772.

$15 \beta, 16 \beta$-亚甲基雄甾-2-(3,4,5-三甲氧基)-苯亚甲基4,6-二烯-3,17-二酮 $(\mathbf{5 j})$ : 黄色固体，产率 73\%. m.p. 226.0 228.1 ${ }^{\circ} \mathrm{C} ;{ }^{1} \mathrm{H}$ NMR (400 MHz, $\left.\mathrm{CDCl}_{3}\right) \delta: 7.66(\mathrm{~d}$, $J=2.7 \mathrm{~Hz}, 1 \mathrm{H}), 6.61(\mathrm{~s}, 2 \mathrm{H}), 6.43(\mathrm{dd}, J=9.7,2.1 \mathrm{~Hz}$, $1 \mathrm{H}), 6.32$ (dd, $J=9.8,2.8 \mathrm{~Hz}, 1 \mathrm{H}), 5.95$ (s, 1H), 3.89 (s, $3 \mathrm{H}), 3.86(\mathrm{~s}, 6 \mathrm{H}), 2.49 \sim 2.41(\mathrm{~m}, 2 \mathrm{H}), 2.21 \sim 2.10(\mathrm{~m}$, 2H), 1.83 (ddd, $J=8.8,5.3,2.6 \mathrm{~Hz}, 2 \mathrm{H}), 1.74 \sim 1.66(\mathrm{~m}$, $2 \mathrm{H}), 1.52 \sim 1.37(\mathrm{~m}, 3 \mathrm{H}), 1.28 \sim 1.21(\mathrm{~m}, 2 \mathrm{H}), 1.03(\mathrm{~s}$, $6 \mathrm{H}) ;{ }^{13} \mathrm{C} \mathrm{NMR}\left(101 \mathrm{MHz}, \mathrm{CDCl}_{3}\right) \delta: 214.94,188.41$, $161.66,153.07,138.76,136.48,132.71,131.22,128.68$, $124.95,107.03,60.93,56.19,50.80,49.75,42.90,38.25$, $37.80,35.76,35.08,25.99$, 21.57, 20.26, 20.12, 18.58, 17.41; HRMS (ESI) calcd for $\mathrm{C}_{30} \mathrm{H}_{35} \mathrm{O}_{5}[\mathrm{M}+\mathrm{H}]^{+}$ 475.2484, found 475.2485.

$15 \beta, 16 \beta$-亚甲基雄甾-2-(2-甲氧基)-苯亚甲基-4,6-二 烯-3,17-二酮(5k): 淡黄色固体，产率 75\%. m.p. 156.7 $158.2{ }^{\circ} \mathrm{C} ;{ }^{1} \mathrm{H}$ NMR (400 MHz, $\left.\mathrm{CDCl}_{3}\right) \delta: 7.85$ (d, $J=2.8$ $\mathrm{Hz}, 1 \mathrm{H}), 7.36 \sim 7.29(\mathrm{~m}, 1 \mathrm{H}), 7.22(\mathrm{dd}, J=7.5,1.6 \mathrm{~Hz}$, $1 \mathrm{H}), 7.01 \sim 6.90(\mathrm{~m}, 2 \mathrm{H}), 6.40(\mathrm{dd}, J=9.7,2.0 \mathrm{~Hz}, 1 \mathrm{H})$, $6.30(\mathrm{dd}, J=9.8,2.7 \mathrm{~Hz}, 1 \mathrm{H}), 5.94$ (s, 1H), 3.84 (s, 3H),
$2.45 \sim 2.36(\mathrm{~m}, 2 \mathrm{H}), 2.14(\mathrm{ddd}, J=15.7,9.5,4.4 \mathrm{~Hz}, 2 \mathrm{H})$, $1.82(\mathrm{ddd}, J=8.8,5.4,2.8 \mathrm{~Hz}, 1 \mathrm{H}), 1.79 \sim 1.73(\mathrm{~m}, 2 \mathrm{H})$, $1.70(\mathrm{td}, J=5.2,2.9 \mathrm{~Hz}, 1 \mathrm{H}), 1.40(\mathrm{ddd}, J=21.5,10.0,6.9$ $\mathrm{Hz}, 3 \mathrm{H}), 1.27 \sim 1.21(\mathrm{~m}, 2 \mathrm{H}), 1.01(\mathrm{~d}, J=3.2 \mathrm{~Hz}, 6 \mathrm{H}) ;{ }^{13} \mathrm{C}$ NMR (101 MHz, $\left.\mathrm{CDCl}_{3}\right) \delta: 215.24,188.72,161.64$, $158.27,138.49$, 132.98, 132.72, 129.8, 129.78, 128.77, $125.08,124.84,120.05,110.80,55.50,50.86,49.76,42.93$, $38.36,37.84,35.72,35.02,25.99,21.61,20.17,20.14$, 18.30, 17.43; HRMS (ESI) calcd for $\mathrm{C}_{28} \mathrm{H}_{31} \mathrm{O}_{3}[\mathrm{M}+\mathrm{H}]^{+}$ 415.2273, found 415.2272.

\section{3 生物活性测试}

\subsection{1 小鼠小胶质细胞系 BV2 的培养}

以 DMEM 培养基作为基础配制成含 $10 \%$ (体积分 数)胎牛血清的细胞培养液, BV2 小胶质细胞在 5\%(体积 分数) $\mathrm{CO}_{2}, 37{ }^{\circ} \mathrm{C}$ 培养瓶中传代培养, 至第三天贴壁细胞 约占培养瓶底面积 $70 \% \sim 80 \%$ ，以胰酶消化贴壁细胞， 传代至另一培养瓶. 以复苏后的 BV2 作为第一代, 选择 第 3 8 代 BV2 细胞进行实验.

\subsection{2 药物配制方法}

11 种化合物均为固体状, 用 DMSO 溶解. 配成母 液，浓度为 $100 \mathrm{mmol} \cdot \mathrm{L}^{-1}$ ，储存于 $-20{ }^{\circ} \mathrm{C}$. 临用时用 DMEM 培养液将其进行稀释，依次稀释为 100, 30, 10 ， $1 \mu \mathrm{mol} \cdot \mathrm{L}^{-1}$. DMSO 终浓度 $<1 \%$.

3.3.3 Griess 法检测化合物对 LPS 激活小胶质细胞的 抑制作用

取对数生长期的 BV2 小胶质细胞，用含 $10 \%$ (体积 分数)胎牛血清的新鲜 DMEM 培养基将细胞密度调至 $2.5 \times 10^{5}$ cells $/ \mathrm{mL}$ ，接种于 96 孔板内，100 $\mu \mathrm{L} / \mathrm{well}$, 于 $37{ }^{\circ} \mathrm{C}, 5 \% \mathrm{CO}_{2}$ 的培养箱内培养. 细胞贴壁培养 $24 \mathrm{~h}$ 后 换成无血清的新鲜培养液, 同时进行加药处理. 11 种化 合物设剂量 $100,30,10,1 \mu \mathrm{mol} \cdot \mathrm{L}^{-1}$ 与 LPS 共同作用. 同 时设空白对照. 各给药组中 LPS 终浓度为 $100 \mathrm{ng} / \mathrm{mL}$. 细胞加药后继续培养 $24 \mathrm{~h}$ 后, 收集上清液, Griess 比色 法检测上清液中 NO 含量.

3.3.4 MTT 法检测化合物对小胶质细胞细胞成活率 的影响

取对数生长期培养的 BV2 小胶质细胞, 用含 $10 \%$ (体积分数)胎牛血清的新鲜 DMEM 培养基将细胞 密度调至 $2.5 \times 10^{5} \mathrm{cells} / \mathrm{mL}$, 接种于 96 孔板内, 100 $\mu \mathrm{L} /$ well, 于 $37{ }^{\circ} \mathrm{C}, 5 \%$ (体积分数) $\mathrm{CO}_{2}$ 的培养箱内培养. 细胞贴壁培养 $24 \mathrm{~h}$ 后换成新鲜培养液, 同时进行加药处 理. 11 种化合物设剂量 $100,30,10,1 \mu \mathrm{mol} \cdot \mathrm{L}^{-1}$ 与 LPS 共 同作用. 同时设空白对照. 各给药组中 LPS 终浓度为 $100 \mathrm{ng} / \mathrm{mL}$. 细胞加药后继续培养 $24 \mathrm{~h}$, 然后向细胞液中 
加入 MTT 溶液, $10 \mu \mathrm{L} / w e l l$, 将细胞与 $0.25 \mathrm{mg} / \mathrm{mL} \mathrm{MTT}$ 于 $37{ }^{\circ} \mathrm{C}$ 下共同孵育 $3 \mathrm{~h}$, 吸除培养液, 然后加入 $150 \mu \mathrm{L}$ 的 DMSO 溶液, 测定其光密度 OD 值. 如下公式计算细 胞成活率. 细胞成活率 $(\%)=$ 样品组 $\mathrm{OD}$ 值的平均值/空 白对照组 OD 值的平均值 $\times 100 \%$

\subsection{5 统计方法}

全部资料采用 SPSS 统计软件包进行检验分析. 结 果用平均值土标准误表示. 采用 One-Way ANOVA 结合 Dunnett T3 或 LSD 进行统计分析.

辅助材料(Supporting Information) 化合物 3, 4, 5a 5k 的 ${ }^{1} \mathrm{H}$ NMR 和 ${ }^{13} \mathrm{C}$ NMR 谱图. 这些材料可以免费从 本刊网站(http://sioc-journal.cn/)上下载.

\section{References}

[1] Lai, L. M.; Ling, C. X.; Zhang, X. F.; Hu, C. H. J. Shangqiu Teach. Coll. 2018, 34, 27 (in Chinese). (来兰梅, 凌翠霞, 张向飞, 胡春华, 商丘师范学院学报, 2018, $34,27$.

[2] (a) Barger, S. W.; Harmon, A. D. Nature 1997, 388, 878. (b) Chang, J. Y.; Chavis, J. A.; Liu, L. Z.; Drew, P. D. BiochemBiophys. Res. Commun. 1998, 249, 817.

[3] (a) Carlier, P. R.; Chow, E. S.-H.; Han, Y.; Liu, J.; Yazal, J. E.;
Pang, Y.-P. J. Med. Chem. 1999, 42, 4225.

(b) Li, L.; Yan, L.; Xiao, M. Q.; Zhong, C. C.; Rui, X.; Gan, Y. X.; Qing, S.; Zheng, H. T.; Yong, D. Bioorg. Med. Chem. 2017, 25, 1997.

[4] (a) Scherbakov, A. M.; Zavarzin, I. V.; Vorontsova, S. K.; Alakanada, H.; Andreeva, O. E.; Yadykov, A. V.; Levina, I. S.; Volkova, Y. A.; Shirinian, V. Z. Steroids 2018, 138, 91.

(b) Bano, S.; Javed, K.; Ahmad, S.; Rathish, I. G.; Singh, S.; Chaitanya, M.; Arunasree, K. M.; Alam, M. S. Eur. J. Med. Chem. 2013, 65, 51

(c) Liu, H. R.; Zhou, C.; Fan, H. Q.; Tang, J. J.; Liu, L. B.; Gao, X. H.; Wang, Q. A.; Liu, W. K. Chem. Biol. Drug Des. 2015, 86, 517.

[5] Ren, S. T.; Wang, L.; Wu, Y. R.; Liu, X. J.; Wang, Y. X.; Liu, S. H. J. Huaihai Inst. Technol. (Nat. Sci. Ed.) 2018, 27, 32 (in Chinese). (任抒婷，王蕾，吴显然，刘玮炜，刘秀坚，王有宪，刘书豪，淮 海工学院学报(自然科学版), 2018, 27, 32.)

[6] (a) Zhang, X.; Rakesh, K. P.; Bukhari, S. N. A.; Balakrishna, M.; Manukumar, H. M.; Qin, H. L. Bioorg. Chem. 2018, 80, 86.

(b) Lahtchev, K. L.; Batovska, D. I.; Parushev, S. P.; Ubiyvovk, V. M.; Sibirny, A. A. J. Med. Chem. 2008, 43, 2220.

[7] Zhou, L.; Ren, M. L. Int. J. Geriatr. 2003, 24, 256 (in Chinese). (周璘，任慕兰，国际老年医学杂志，2003，24，256.)

[8] Schverer, M.; Lanfumey, L.; Baulieu, E.-E.; Froger, N.; Villey, I. Pharmacol. Ther. 2018, 191, 190 .

[9] Shan, L. H.; Liu, H. M.; Jiang, D. D.; Zhao, S. S.; Qiao, X.; Zhang, L. J. $C N 106591155,2017$.

[10] Romano, A.; Romano, D.; Ragg, E.; Costantinoc, F.; Lennac, R.; Gandolfid, R.; Molinaria, F. Steroids 2006, 71, 429.

[11] Walter, C.; Fablo, B.; Marco, A.; Manuela, R.; Clinzia, B. EP $1903051,2008$. 